Unfallchirurg 2014 $\cdot 117: 582-582$

DOI 10.1007/s00113-014-2569-4

Online publiziert: 18. Juli 2014

(c) Springer-Verlag Berlin Heidelberg 2014

\title{
M. Schultheiss
}

Klinik für Unfall-, Hand-, Plastische und Wiederherstellungschirurgie, Universitätsklinikum Ulm

\section{Interdisziplinäre Behandlung von Knochensarkomen: Teil II}

Sehr geehrte Kolleginnen und Kollegen,

In Heft 6/14 wurden die interdisziplinären Aspekte in der Behandlung von Knochensarkomen dargestellt. Nun soll dieses Leitthema mit dem Fokus auf die operativen Techniken fortgesetzt werden.

Die Wichtigkeit des Hand-in-HandVorgehens bei der operativen Planung in Einklang mit der bioptischen Maßnahme wurde diesbezüglich bereits thematisiert. Dennoch bleibt die Resektion verbunden mit einer biologischen oder prothetischen Rekonstruktion eine komplikationsbelastete Operation. Zudem stellt sich oft die Frage der Weite und der Notwendigkeit eines ausreichenden Sicherheitsabstands nach neoadjuvanter Chemotherapie.

In diesem Heft werden die Möglichkeiten der Resektion und Rekonstruktion und das Management von möglichen Komplikationen beschrieben. Ferner werden Ihnen Lösungswege für spezielle Situationen, wie die der pathologischen Fraktur offeriert.

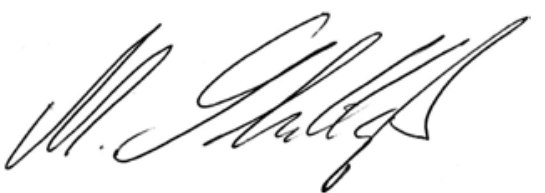

M. Schultheiss



Interessenkonflikt. M. Schultheiss gibt an, dass kein Interessenkonflikt besteht. 